\title{
Multi-interactive coordination network featuring a ligand with topologically isolated $p$-orbitals
}

\author{
Y. Wada, Hiroyoshi Ohtsu, Pavel M. Usov, Masaki Kawano \\ Department of Chemistry, School of Science, Tokyo Institute of Technology \\ mkawano@chem.titech.ac.jp
}

We reported multi-interactive ligand, 2,5,8-tri(4'-pyridyl)-1,3,4,6,7,9-hexaazaphenalenate (4-TPHAP') with topologically isolated porbitals on an interactive HAP skeleton, can trap metastable states via intermolecular interactions during network formation. ${ }^{1,2}$ Kinetic assembly of porous coordination networks create interactive sites in the pore, ${ }^{3}$ which can trap guest molecules and visualize their structure, for example small reactive sulphur allotropes, and conversion. ${ }^{4}$

In this study, a lower symmetry derivative of the HAP ligand containing 3-pyridyl groups (3-TPHAP-) was developed. The purpose of this ligand was to obtain network structures with minimized dynamic motion compared to 4-TPHAP- based networks. In 3-TPHAP', the pyridine ring rotation becomes suppressed after coordination to a metal centre. The lack of rotational motion may significantly influence the guest encapsulation behaviour in the resultant structures. This ligand was prepared by a one-pot condensation reaction. It was successfully reacted with a $\mathrm{Co}^{2+}$ salt and 1,4-benzenedicarboxylic acid co-ligand to give a porous coordination network. In the structure, HAP skeleton interacts with water to form an internal hydrogen bonding network, allowing to reveal the entire pore space by single crystal X-ray diffraction (SXRD). The network structure consists of dimeric Co clusters featuring labile sites occupied by solvent molecules. Several guest molecules, namely anthracene, triphenylene and iodine, were incorporated inside the network. The resultant encapsulated structures were elucidated by SXRD revealing unusual host-guest interactions with a subtle structure modulation.

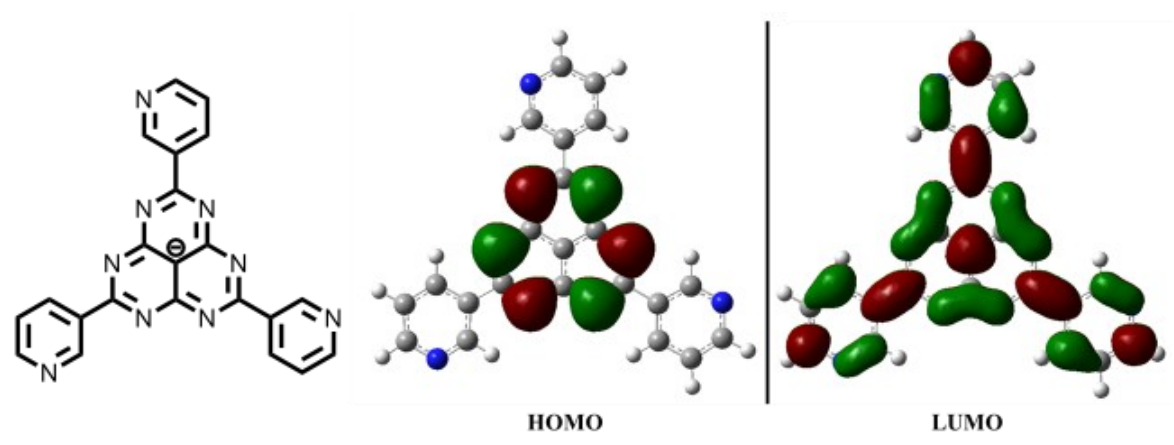

Figure 1. The chemical structure of 3-TPHAP-, showing its HOMO and LUMO.

[1] Y. Yakiyama, A. Ueda, Y. Morita, M. Kawano, Chem. Commun., 2012, 48, 10651.

[2] T. Kojima, T. Yamada, Y. Yakiyama, E. Ishikawa, Y. Morita, M. Ebihara, M. Kawano, CrystEngComm, $2014,16,6335$.

[3] (a) H. Kitagawa, H. Ohtsu, M. Kawano, Angew. Chem. Int. Ed., 2013, 52, 12395. (b) H. Ohtsu, M. Kawano, Chem. Commun. $2017,53,8818$.

[4] H. Kitagawa, H. Ohtsu, A. J. Cruz-Cabeza, M. Kawano, IUCrJ, 2016, 3, 232.

Keywords: Porous coordination networks; Multi-interactive ligand; Guest encapsulation 